\title{
Further results on controllability of linear systems with switching delays ${ }^{\star}$
}

\author{
Raphaël M. Jungers* Alessandro D'Innocenzo** \\ Maria D. Di Benedetto ** \\ * ICTEAM Institute, Université catholique de Louvain, \\ Louvain-la-Neuve, Belgium. Email: raphael.jungers@uclouvain.be \\ ** Center of Excellence DEWS, Department of Information \\ Engineering, Computer Science and Mathematics, University of \\ L'Aquila, Italy. Email: alessandro.dinnocenzo@univaq.it, \\ mariadomenica.dibenedetto@univaq. it
}

\begin{abstract}
We prove two more results on controllability of LTI systems with switching delays. Firstly, we improve our previous results on controllability verification (when arbitrarily large look-ahead of the switching signal is allowed) by providing an algorithm which is not restricted to regular matrices. In particular we provide a more detailed analysis for nihilpotent matrices (i.e. matrices with only zero eigenvalues): we restate this problem as a completely combinatorial one and provide a polynomial time algorithm, which has much better efficiency than a naive brute force algorithm.

Secondly, we show that when the switching signal cannot be measured it can be necessary to use nonlinear controllers for stabilizing a linear plant.
\end{abstract}

\section{INTRODUCTION}

Wireless Networked Control Systems are spatially distributed control systems where the communication between sensors, actuators, and computational units is supported by a wireless multi-hop communication network. The main motivation for studying such systems is the emerging use of wireless technologies for control systems (see e.g. Akyildiz and Kasimoglu [2004], Han et al. [2010] and references therein) and the recent development of wireless industrial control protocols (such as WirelessHART and ISA-100). Although the use of wireless networked control systems offers many advantages with respect to wired architectures (e.g. flexible architectures, reduced installation/debugging/diagnostic/maintenance costs) their use is a challenge when one has to take into account the joint dynamics of the plant and of the communica-

\footnotetext{
* The research leading to these results has received funding from the European Union Seventh Framework Programme [FP7/2007-2013] under grant agreement n257462 HYCON2 Network of excellence. R.M.J. is supported by the Communauté francaise de Belgique Actions de Recherche Concertées, and by the Belgian Programme on Interuniversity Attraction Poles initiated by the Belgian Federal Science Policy Office. R.J. is a F.R.S.-FNRS Research Associate.
}

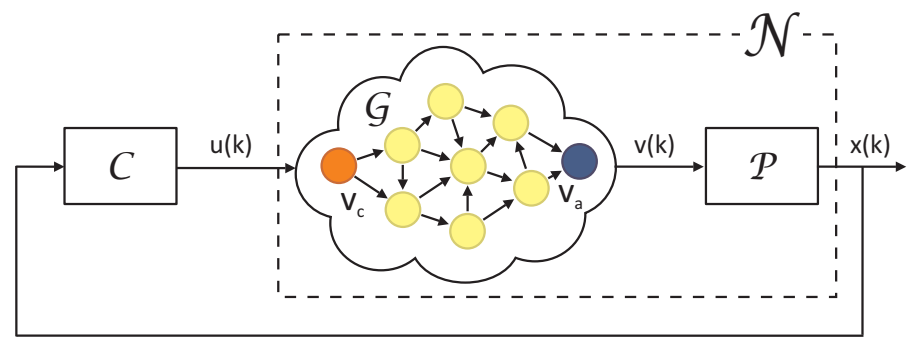

Fig. 1. State feedback networked control scheme. tion protocol. Recently, a huge effort has been made in scientific research on Networked Control Systems (NCS), see e.g. Zhang et al. [2001], Walsh and Ye [2001], Antsaklis and Baillieul [2004], Andersson et al. [2005], Gupta et al. [2009], Hespanha et al. [2007],Heemels et al. [2010]) and on the interaction between control systems and communication protocols (see e.g. Aström and Wittenmark [1997], Walsh et al. [2002], Yook et al. [2002], Tabbara and Nešić [2007], Tabbara et al. [2007]). In general, the literature on NCSs addresses non-idealities (e.g. quantization errors, packets dropouts, variable sampling and delay, communication constraints) as aggregated network performance variables, neglecting the dynamics introduced by the communication protocols. In Andersson et al. [2005], , a simulative environment of computer nodes and communication protocols interacting with the continuous-time dynamics of the real world is presented. To the best of our knowledge, the first integrated framework for analysis and co-design of network topology, scheduling, routing and control in a wireless multi-hop control network has been presented in Alur et al. [2011], where switching systems are used as a unifying formalism for control algorithms and communication protocols, and in extensions of this framework D'Innocenzo et al. [2013], Pajic et al. [2011]. Following this research line, we restrict our focus in this paper on the effect of routing and consider a multi-hop network $\mathcal{G}$ that provides the interconnection between a state-feedback discrete-time controller $\mathcal{C}$ and a discretetime LTI plant $\mathcal{P}$ (see Figure 1 ). The network $\mathcal{G}$ consists of an acyclic graph where the node $v_{c}$ is directly connected to the controller and the node $v_{u}$ is directly connected to the actuator of the plant. As classically done in (wireless) multi-hop networks to improve robustness with respect to node failures we exploit redundancy of routing paths, namely we assume that the number of paths 
that interconnect $\mathcal{C}$ to $\mathcal{P}$ is greater than one and that for any actuation data sent from the controller to the plant a unique path is chosen. Each path is characterized by a delay in forwarding the data (see D'Innocenzo et al. [2013] for details), as a consequence each actuation data will be delayed of a finite number of time steps according to the chosen routing. Since this choice usually depends on the internal status of the network, i.e. because of node and/or link failures, we consider the choice of the routing path as an external disturbance and address both the cases when it is measurable or not. Hence, we obtain as a model a particular sort of switching system, where the switching signal describes the evolution of the delay with respect to time.

Systems with time-varying delays have attracted increasing attention in recent years (see e.g. Liu et al. [2006], Hetel et al. [2007], Heemels et al. [2010], Shao and Han [2011], Lombardi et al. [2012] and references therein). In Hetel et al. [2011] it is assumed that the time-varying delay is approximatively known and numerical methods are proposed to exploit this partial information for adapting the control law in real time. Our model differs from this setting because due to the routing, control commands generated at different times can reach the actuator simultaneously, their arrival time can be inverted, and it is even possible that at certain times no control commands arrive to the actuator.

In another line of research, sufficient stability conditions and LMI based design procedures have been developed for discrete-time switching systems with time-varying delays (see e.g. L. Hetel and Iung [2006] and Zhang et al. [2008]). These methods do not take into account the specific structure of the systems induced by the fact that the switching is restricted on the delay-part of the dynamics. Our goal is to leverage this particular structure in order to improve our theoretical understanding of the dynamics at stake in these systems. As we will see, it enables us to design tailored controllers whose performances or guarantees are better than for classical switching systems.

In a previous work Jungers et al. [2012] we proved that our time-varying delay systems can be represented by pure switching systems, where the switching matrices assume a particular form. Switching systems are notably hard to control, and one often has to resort to Lyapunovlike conservative techniques (often based on LMI formulations) in order to handle them. However we show that the characteristic structure of these systems allows for a more analytical understanding of the dynamics, leading to exact algorithms for some particular questions. We think that these dynamics, lying at the frontier between easy LTI systems and hard (and often untractable) switching systems are interesting both for theoretical and applied purposes.

We will address the controller design problem by assuming that, for each time $t$, the controller is aware of the $N \geq 0$ next future routing path choices. If $N=0$ the controller is not aware of any of the past, current and future propagation delays: we will call this situation the delay independent case. If $N \geq 1$ the controller is aware of the propagation delays of the actuation signals sent at times $t, t+1, \ldots, t+N-1$, and keeps memory of the past delays: we will call this situation the delay dependent case. We call $N$ the look-ahead parameter.

From the network point of view the practically admissible values for $N$ depend on the protocol used to route data (see Y. Yang and Kravets [2005] and references therein for an overview on routing protocols for wireless multihop networks). If the controller node $v_{c}$ of $\mathcal{G}$ is allowed by the protocol to chose a priori the routing path (e.g. source routing protocols), then we can assume that the controller is aware of the routing path and the associated delay, and therefore is also aware of the switching signal (i.e. $N>0$ ). If instead the protocol allows each communication node to choose the next destination node according to the local neighboring network status information (e.g. hop-byhop routing protocols) then we cannot assume that the controller is aware of the routing path, and therefore of any of the past, current and future propagation delays (i.e. $N=0)$.

We addressed the corresponding modeling and stability verification problems in Jungers et al. [2012], while in Jungers et al. [2014] we tackled the harder controller design problem for the delay dependent case providing an algorithm for controllability verification restricted to regular plant matrices.

In this work we first analyze the situation where one can chose an arbitrarily large (but finite) look-ahead parameter $N$. This can occur in situations where the dynamics of the plant is given, but one can design the protocol, and thus require a certain look-ahead knowledge if needed. We prove that in this case the controllability verification problem can be split into two sub-problems, one characterized by a regular matrix and one by a nihilpotent matrix: since the regular case has been already solved in Jungers et al. [2014], we address here the nihilpotent case and provide a polynomial time algorithm.

In the second part of the paper, we analyze the situation where the look-ahead parameter $N$ is fixed and given as part of the problem. We show that in this case it is much harder to decide controllability of the plant: indeed we show that it can be necessary to make use of nonlinear controllers for stabilizing a linear plant.

The paper is organized as follows: in Section 2 we quickly recall the problem formulation and the modeling framework from Jungers et al. [2012]. In Sections 3 and 4 we address the controller design problem for the arbitrarily large look-ahead and the fixed look-ahead cases. In Section 5 we conclude and propose open problems for future research.

The proofs are omitted due to length constraints.

\section{MODELING}

In this paper we will address the problem of stabilizing a discrete-time LTI system of the form

$$
x(t+1)=A x(t)+B u(t), \quad y(t)=x(t), \quad t \geq 0,
$$

with $A \in \mathbb{R}^{n \times n}$ and $B \in \mathbb{R}^{n \times m}$, using a state-feedback controller $\mathcal{C}$. From now on we will obviously assume that the plant pair $(A, B)$ is controllable. We assume that the control signal $v(t)$ generated by $\mathcal{C}$ is relayed to the actuator of the plant $\mathcal{P}$ via a multi-hop network Alur et al. [2011]. The network $\mathcal{G}$ consists of an acyclic graph $(V, E)$, 
where the node $v_{c} \in V$ is directly interconnected to the controller $\mathcal{C}$ and the node $v_{u}$ is directly interconnected to the actuator of the plant $\mathcal{P}$. Since the network exploits redundancy of routing paths the number of paths that can be used to reach $v_{u}$ from $v_{c}$ is assumed to be greater than one. In order to relay each actuation data $v(t)$ to the plant, at each time step $t$ a unique path of nodes that starts from $v_{c}$ and terminates in $v_{u}$ is exploited. To each path a different delay can be associated in transmitting data from $v_{c}$ to $v_{u}$, depending on the transmission scheduling and on the number of hops to reach the actuator (see D'Innocenzo et al. [2013] for details). Since the choice of the routing path usually depends on the internal status of the network we assume that the chosen routing path is time-varying. As a consequence the control signal $v(t)$ at time $t$ will be delayed of a finite number of time steps that we model as a disturbance signal $\sigma(t) \in D: t \geq 0$, where $D \subseteq\left\{0,1, \ldots, d_{\max }\right\}$ is the set of possible delays introduced by all routing paths and $d_{\max }$ is the maximum delay. For the reasons above we model the dynamics of the networked control system $\mathcal{N}$ as follows ${ }^{1}$ :

Definition 1. The dynamics of the interconnected system $\mathcal{N}$ can be modeled as

$$
\begin{aligned}
x(t+1) & =A x(t)+B u\left(v\left(t-d_{\max }: t\right), \sigma\left(t-d_{\max }: t\right)\right) \\
& =A x(t)+B \sum_{\substack{t-d_{\max } \leq t^{\prime} \leq t \\
t^{\prime}+\sigma\left(t^{\prime}\right)=t}} v\left(t^{\prime}\right),
\end{aligned}
$$

where $v\left(t-d_{\max }: t\right), \sigma\left(t-d_{\max }: t\right)$ represents $^{2}$ the latest $d_{\max }+1$ values of the output of the controller $v(\cdot) \in \mathbb{R}^{m}$ and of the switching signal $\sigma(\cdot) \in D$. We define the signal of actuation times $\tau: \mathbb{N} \rightarrow\{0,1\}$ such that $\tau(t)=1$ if there exists $t^{\prime} \leq t$ with $t=t^{\prime}+\sigma\left(t^{\prime}\right), \tau(t)=0$ otherwise.

We now define the control signal $v$ as a function of its inputs. In order to keep things simple, we suppose that the controller measures the full state $x(t)$. As said above, on top of this, at each time $t$, the controller is aware of the $N \geq 0$ future routing path choices, and therefore of the future propagation delays $\sigma(t), \sigma(t+$ $1), \ldots, \sigma(t+N-1)$ (that is, if $N=0$ the controller does not know any propagation delay). We also assume that the controller keeps memory of the past $d_{\max }$ switching signals $\sigma\left(t-d_{\max }\right), \ldots, \sigma(t-1)$. For classical (i.e. non-switched) feedback systems with fixed delay it is well known that the system can be neither controllable nor stabilizable if the feedback only depends on $x(t)$, that is if the controller does not have a memory of its past outputs. Therefore, we also assume that the controller keeps memory of its past $d_{\max }$ outputs $v\left(t-d_{\max }\right), \ldots, v(t-1)$.

As a consequence we can assume without loss of generality that, in the delay-dependent case $(N>0)$, the output signal $v$ of the controller depends on its past $d_{\text {max }}$ output values, on the current plant state, on the past $d_{\max }$ switching signals and on the future $N$ switching signals, namely:

$$
v\left(t, v\left(t-d_{\max }: t-1\right), x(t), \sigma\left(t-d_{\max }: t+N-1\right)\right),
$$

\footnotetext{
1 Of course, one can expand the statespace in order to represent delays, since it is finite-dimensional. However, this is not useful for our purpose, so we chose a more concise representation.

2 This is the "matlab notation".
}

because this information encapsulates the full state of the closed-loop system.

In the delay-independent case $(N=0)$ this is not possible since the controller ignores the current and thus also the previous values of the switching signal, and the only variables it can use are the past values of $x(t)$ and of the control commands, namely ${ }^{3}$ :

$$
v\left(t, x\left(t-d_{\max }: t\right), v\left(t-d_{\max }: t-1\right)\right) .
$$

Remark 1. The above model is quite general and allows representing a wide range of routing communication protocols for (wireless) multi-hop networks Y. Yang and Kravets [2005]. We remark that several variations are possible. For instance, in our setting, it could happen during the run of the system that at some particular time no feedback signal comes back to the plant: we assume that in this situation the actuation input to the plant is set to zero. A variation is to implement a hold that would keep memory of the previous input signal and resend it if the new one is empty. Moreover, in our setting, it could also happen that two control signals sent at different times reach the actuator simultaneously: we assume that in this situation the actuation input to the plant is set to the sum of the control signals that arrive simultaneously. A variation is to keep the most recent control signal and discard all the others. We defer the comparison of such variants for further studies (see Gommans et al. [2013] for a recent work that provides a comparison among some of these approaches and also takes into account packet dropouts).

\section{DELAY DEPENDENT CONTROLLER DESIGN}

In this section we address the following problem: given a system with variable delays as in (1), does there exist a sufficiently large look-ahead $N$, together with a particular controller, such that the closed loop system is controllable for any switching signal? Very little is known in the literature about the controllability of switching systems, and thus this question is quite challenging.

We restrict ourself to the single input case, i.e. $m=1$. Thus, $B$ is a vector, which we denote by $b$ for the sake of clarity. The extension to $m>1$ is not straightforward, and will be the topic of further work. We first define the controllability notion for System (1).

Definition 2. We say that the system (1) is controllable with look-ahead $N$ if, for any initial state $x(0)=x_{0}$, any final state $x_{f}$ and any switching signal $\sigma(t)$, there exists a control signal $v\left(t, v\left(t-d_{\max }: t-1\right), x(t), \sigma\left(t-d_{\max }: t+\right.\right.$ $N)$ ) such that

$$
\exists t \geq 0: x(t)=x_{f} .
$$

If there is no look-ahead $N$ such that the system is controllable, we say that the system is uncontrollable.

The existence of a controller as in Definition 2 seems to be very hard to decide: to the best of our knowledge, controller design is widely overlooked in the literature on general switching systems, and only sufficient conditions for the existence of a linear controller are known Ge and Sun [2005], Lee and Dullerud [2006]. As a first attempt

\footnotetext{
3 We arbitrarily restrict the memory of the controller to $d_{\max }$. We defer to further research the impact of the size of the memory on the performances of the controller in this case.
} 
to tackle the problem for our systems with varying delays, we assume here that the controller can require an arbitrary look-ahead knowledge of the switching signal. However, as one will see below, this is in some cases not sufficient to ensure controllability, even if the pair $(A, b)$ is controllable. Nevertheless, it turns out that it is possible to decide controllability, and efficiently build a controller in the controllable cases. We will show that one can compute a finite value $N$ depending only on the dimension of the plant and the set of delays such that if the system is controllable with infinite look-ahead, it is controllable with finite look-ahead $N$. This obviously gives conditions for controllability when the look-ahead is a fixed finite value $N^{\prime}$ : if the system is uncontrollable with infinite look-ahead, it is clearly uncontrollable with the actual value $N^{\prime}$; if, on the other hand, the system is controllable with infinite look-ahead and $N^{\prime} \geq N$, then the actual system is controllable. In the next definition, we define a controllability matrix as if the controller knew at time $t=0$ the infinite future sequence of signals $\sigma(t)$.

Definition 3. Given a system (1) and a switching signal $\sigma(t)$ we define

$$
C_{t}(A, B, D, \sigma(t))
$$

the controllability matrix at time $t$, whose columns are given by

$$
\left\{A^{t-t^{\prime}-\sigma\left(t^{\prime}\right)} B: t^{\prime} \geq 0, t-t^{\prime}-\sigma\left(t^{\prime}\right) \geq 0\right\} .
$$

The order of the columns (although not that important) is by increasing order of $t^{\prime}$, so that

$$
x(t)=A^{t} x_{0}+C_{t} v_{t},
$$

where the components of $v_{t}$ are given by

$$
v_{t}\left(t^{\prime}\right)=v\left(t^{\prime}\right): t^{\prime} \geq 0, t-t^{\prime}-\sigma\left(t^{\prime}\right) \geq 0,
$$

namely by all the control signals delivered to the actuator up to time $t$.

We denote by $\operatorname{span}\left(C_{t}\right)$ the space generated by the columns of the matrix $C_{t}$.

Proposition 1. The system (1) is uncontrollable if and only if there exists a switching signal $\sigma(t)$ such that, for all $t \in \mathbb{N}$, we have

$$
\operatorname{span}\left(C_{t}\right) \neq \mathbb{R}^{n} \text {. }
$$

In Jungers et al. [2014] we provided an exponential time algorithm for controllability verification (when arbitrarily large look-ahead of the switching signal is allowed) which is restricted to regular matrices $A$ of the plant. In this work we first prove that the controllability verification problem can always be split into two sub-problems, one characterized by a regular matrix and one by a nihilpotent matrix: since the regular case has already been solved in Jungers et al. [2014], the end of the section is devoted to an algorithm which works for nihilpotent matrices. In fact, in case the matrix only has zero eigenvalues (i.e. if it is nihilpotent), our algorithm even terminates in polynomial time.

\subsection{Reduction to nihilpotent matrices}

Lemma 1. If the matrix $A$ has more than one Jordan block with eigenvalue zero, the system is uncontrollable.

Lemma 2. Suppose that the matrix $A$ has one Jordan block of size $k$ with eigenvalue zero. That is, there is an invertible matrix $T$ such that

$$
T A T^{-1}=\left(\begin{array}{cc}
J_{0, k} & 0 \\
0 & A^{\prime}
\end{array}\right), \quad T b=\left(\begin{array}{c}
b_{0} \\
b^{\prime}
\end{array}\right) .
$$

Then, the system (1) is uncontrollable if and only if the system $\left(J_{0, k}, b_{0}\right)$ is uncontrollable with the set of delays $D$, or the system $\left(A^{\prime}, b^{\prime}\right)$ is uncontrollable with the set of delays $D$.

\subsection{A naive algorithm for nihilpotent matrices}

Since the case of a regular matrix has been tackled in Jungers et al. [2014], we are thus left with the problem of deciding whether a system with a controllable pair $\left(J_{0, k}, b\right)$ is uncontrollable with a given set of delays $D$. It turns out that there is a very simple combinatorial characterization of the controllability matrix to be full rank in that case:

Lemma 3. Suppose that the matrix $A$ is a single Jordan block of size $k$ with eigenvalue zero. Then, the controllability matrix is full rank if and only if $b, A b, A^{2} b, \ldots A^{k-1} b$ are columns of the controllability matrix.

Thus, the controllability problem for nihilpotent matrices amounts to check whether there exists a sequence of delays $\sigma: \mathbb{N} \rightarrow D$ such that the controllability matrix never contains $b, A b, A^{2} b, \ldots, A^{k-1} b$. In the next subsection we derive an efficient (polynomial time) procedure for that. In this subsection we quickly show that there is a simple exponential time procedure to answer this question. The reason is that the polynomial-time algorithm that we derive in the next subsection is exclusively designed for the particular problem at stake here (i.e. one input vector $b$, a single Jordan block $A=J_{0, k}$ ) while we believe that the exponential time algorithm we derive here can be used in more general contexts.

Lemma 4. Suppose that the matrix $A$ is a single Jordan block of size $k$ with eigenvalue zero. Then, the rank of the controllability matrix at time $t$ only depends on the values of the switching signal at times $t-d_{\max }-k+1, \ldots, t$.

We can now prove the following theorem:

Theorem 1. Let $A, b, D$ represent a system as in (1). If $A$ is a nihilpotent matrix, a naive algorithm to verify controllability takes $O\left(\left(d_{\max }+k\right)|D|^{d_{\max }+k}\right)$ operations.

\subsection{A polynomial time algorithm for the nihilpotent case}

We show that when the matrix is nihilpotent there is a polynomial time algorithm to decide controllability. In fact, we are able to characterize the uncontrollable systems as follows:

Theorem 2. Let $A, b, D$ represent a system as in (1). If $A$ is a nihilpotent matrix, then the system is uncontrollable, except if $A$ is (similar to) a $2 \times 2$ Jordan block and the set of delays has only two different delays, of equal parity. In that case the minimal look-ahead needed to guarantee controllability is equal to $d_{\max }$.

Corollary 1. If $A$ is a nihilpotent matrix, there is a polynomial time algorithm to decide whether System (1) is controllable with arbitrarily large lookahead.

The following corollary is directly implied by the above corollary and by the results in Jungers et al. [2014]. 
Corollary 2. Given any $A, b, D$ there is an algorithm to decide in finite time whether the system is controllable with arbitrarily large lookahead.

Remark 2. In practice our algorithm for nihilpotent matrices is a constant time algorithm: we basically provide a characterization of the pathological uncontrollable situations, and the algorithm can check these conditions in essentially constant time, independently of the state space dimension or the precise values of the delays.

\section{DELAY INDEPENDENT CONTROLLER DESIGN}

When the controller cannot chose the look-ahead the design problem is trickier. In particular, when the lookahead is zero, one has to design a single controller that would work for any possible switching signal. In this section we make some initial steps to tackle the design problem in the delay independent case, and present an example for which there is a nonlinear controller which works better than any linear controller. An asset of our nonlinear controller is that it can detect at time $t+1$ the switching signal $\sigma(t)$ using the state-space measure $x(t+1)$ as a proxy, and make use of it in order to improve the next control signals. Our result implies that restricting oneself to linear controllers leads to conservativeness. This is, to our knowledge, the first example of that kind. It shows that, contrary to what has been done until now in the switching systems literature, one should not restrict his search to linear controllers in order to avoid conservativeness.

Example 1. The system in this example is a rotation of an angle $\alpha$ :

$$
A=\left(\begin{array}{cc}
\cos (\alpha) & -\sin (\alpha) \\
\sin (\alpha) & \cos (\alpha)
\end{array}\right), \quad B=\left(\begin{array}{l}
1 \\
0
\end{array}\right) .
$$

The set of delays is $D=\{0,1\}$, and the controller is delay-independent. We show in the next theorem that this system is stabilizable with a rate of decay equal to $0.69 \ldots$, but no linear controller can achieve a rate smaller than $0.755 \ldots$. .

Theorem 3. For the system from Example 1, there are values of $\alpha$ such that if the system is stabilized with a linear controller, one cannot guarantee the existence of a constant $K$ such that

$$
x(t) \leq K \rho^{t}
$$

for any $\rho<0.755 \ldots$ However, there is a nonlinear controller that allows such a guarantee with $\rho=0.69 \ldots$.

\section{CONCLUSION}

Motivated by applications in wireless control networks, we continue in this paper a research line where we introduced and analyzed a new model of linear time invariant (LTI) systems with switching delays. These systems can be represented as particular switching systems, but in terms of difficulty, they seem to lie halfway between easy LTI systems, for which closed form formulas or efficient algorithms are available for most control questions, and switching systems, for which even the simplest questions are intractable, or bound to conservative solutions.

Contrary to what has been done in the recent literature, our goal was not to apply or adapt switching systemsoriented techniques to our systems, but rather to develop methods that would take advantage of the algebraic structure of these particular switching systems.

We have provided an analytical characterization of controllable systems when enough delays are known in advance. This condition is more complex than for LTI systems, but yet, allows for an algebraic characterization of controllable systems.

On the contrary, we have shown that in general, unlike LTI systems, ours can need nonlinear strategies in order to be stabilized, if there is a limit on the number of forthcoming delays that the controller can know in advance. We believe that this type of results will be useful in forthcoming applications, where the design of the controller and of the communication protocol (i.e. the routing policy) are tangled, as for instance in a multi-hop networked control system.

On top of their practical usefulness, we believe that the questions we investigate are promising for theoretical research, as they necessitate new theoretical ideas in order to answer classical control questions, but yet, they seem to allow for closed form characterizations and algorithmic decision procedures (as opposed to Lyapunov-like methods, which are prevalent for switching systems and are often bound to conservativeness and lack of structure). Our future research will focus on characterizing the computational complexity of the controllability verification problem and algorithmic optimal design of non-linear controllers.

\section{REFERENCES}

I. F. Akyildiz and I. H. Kasimoglu. Wireless sensor and actor networks: Research challenges. Ad Hoc Networks, 2(4):351-367, 2004.

Rajeev Alur, Alessandro D'Innocenzo, Karl Henrik Johansson, George J. Pappas, and Gera Weiss. Compositional modeling and analysis of multi-hop control networks. IEEE Trans. Automat. Contr., 56(10):23452357, 2011.

M. Andersson, D. Henriksson, A. Cervin, and K. Arzen. Simulation of wireless networked control systems. In Proc. of the 44 $4^{\text {th }}$ CDC-ECC, pages 476-481, 2005.

P. Antsaklis and J. Baillieul. Guest editorial special issue on networked control systems. IEEE Trans. on Automatic Control, 49(9):1421-1423, 2004.

K. Aström and B. Wittenmark. Computer-Controlled Systems: Theory and Design. Prentice Hall, 1997.

A. D'Innocenzo, M.D. Di Benedetto, and E. Serra. Fault tolerant control of multi-hop control networks. IEEE Trans. on Automatic Control, 58(6):1377-1389, 2013.

S.S. Ge and Z. Sun. Switched linear systems: Control and design. Communications and control engineering series. Springer, 2005. ISBN 9781852338930. URL http:// books .google.ie/books?id=F\_aVFriAdroC.

T.M.P. Gommans, W.P.M.H. Heemels, N. Bauer, and N. van de Wouw. Compensation-based control for lossy communication networks. International Journal of Control, 86(10):1880-1897, 2013.

V. Gupta, A. F. Dana, J. P. Hespanha, R. M. Murray, and B. Hassibi. Data transmission over networks for estimation and control. IEEE Trans. on Automatic Control, 54(8):1807-1819, 2009. 
S. Han, Z. Xiuming, K. M. Aloysius, M. Nixon, T. Blevins, and D. Chen. Control over wirelesshart network. In Proc. of IECON, pages 2114-2119, 2010.

W.P.M.H. Heemels, A.R. Teel, N. van de Wouw, and D. Nešić. Networked control systems with communication constraints: Tradeoffs between transmission intervals, delays and performance. IEEE Trans. on Automatic Control, 55(8):1781-1796, 2010.

J. P. Hespanha, P. Naghshtabrizi, and Y. Xu. A survey of recent results in networked control systems. Proceedings of the IEEE, 95(1):138-162, 2007.

L. Hetel, J. Daafouz, and C. Iung. Stability analysis for discrete time switched systems with temporary uncertain switching signal. In Proc. of the $46^{t h} C D C$, pages 5623-5628, 2007.

L. Hetel, J. Daafouz, J.-P. Richard, and M. Jungers. Delay-dependent sampled-data control based on delay estimates. Systems and Control Letters, 60(2):146-150, 2011.

R. M. Jungers, A. D'Innocenzo, and M. D. Di Benedetto. Feedback stabilization of dynamical systems with switched delays. Proceedings of the IEEE CDC2012, pages 1325-1330, 2012.

R. M. Jungers, A. D'Innocenzo, and M. D. Di Benedetto. How to control linear systems with switching delays? In Proc. of the $13^{\text {th }} E C C, 2014$. Available from http://perso.uclouvain.be/raphael.jungers/ publis_dispo/jungers_ecc14.pdf.

J. Daafouz L. Hetel and C. Iung. Stabilization of arbitrary switched linear systems with unknown time-varying delays. IEEE Trans. on Automatic Control, 51(10): 1668-1674, 2006.

J.W. Lee and G.E. Dullerud. Uniform stabilization of discrete-time switched and Markovian jump linear systems. Automatica, 42(2):205-218, 2006.

X. G. Liu, R. R. Martin, M. Wu, and M. L. Tang. Delaydependent robust stabilisation of discrete-time systems with time-varying delay. IEE Proc. on Control Theory Appl., 153(6):689-702, 2006.

W. Lombardi, S. Olaru, S.-I. Niculescu, and L. Hetel. A predictive control scheme for systems with variable timedelay. International Journal of Control, 85(7):915-932, 2012.

M. Pajic, S. Sundaram, G. J. Pappas, and R. Mangharam. The wireless control network: a new approach for control over networks. IEEE Trans. on Automatic Control, 56 (10):2305-2318, 2011.

H. Shao and Q.-L. Han. New stability criteria for linear discrete-time systems with interval-like time-varying delays. IEEE Trans. on Automatic Control, 56(3):619-625, 2011.

M. Tabbara and D. Nešić. Input-to-state \& input-output stability of networked control systems. In Proc. of the $46^{\text {th }} C D C$, pages 3321-3326, 2007.

M. Tabbara, D. Nešić, and A. R. Teel. Stability of wireless and wireline networked control systems. IEEE Trans. on Automatic Control, 52(7):1615-1630, 2007.

G. C. Walsh and H. Ye. Scheduling of networked control systems. IEEE Control Systems Magazine, 21(1):57-65, 2001.

G. C. Walsh, H. Ye, and L. G. Bushnell. Stability analysis of networked control systems. IEEE Trans. on Control Systems Technology, 10(3):438-446, 2002.
J. Wang Y. Yang and R. Kravets. Designing routing metrics for mesh networks. In Proc. of the $1^{\text {st }}$ WiMesh, 2005.

J. K. Yook, D. M. Tilbury, N. R. Soparkar, E. Syst, and E. S. Raytheon. Trading computation for bandwidth: Reducing communication in distributed control systems using state estimators. IEEE Trans. on Control Systems Technology, 10(4):503-518, 2002.

L. Zhang, P. Shi, and M. Basin. Robust stability and stabilisation of uncertain switched linear discrete timedelay systems. IET Control Theory and Applications, 2 (7):606-614, 2008.

W. Zhang, M.S. Branicky, and S.M. Phillips. Stability of networked control systems. IEEE Control Systems Magazine, 21(1):84-99, 2001. 